Schmerz 2022 - 36:19-29

https://doi.org/10.1007/s00482-021-00596-9

Angenommen: 27. August 2021

Online publiziert: 18 . Oktober 2021

๑) Der/die Autor(en) 2021

\section{Wahrnehmungen zum Umgang mit Opioiden bei COVID-19}

\section{Eine Umfrage unter Mitgliedern der Deutschen Gesellschaft für Palliativmedizin}

Hintergrund: Obwohl Opioide wirksam Schmerzen und Dyspnoe lindern, findet dies in Leitlinien zur Symptomkontrolle unterschiedliche Gewichtung. Dies kann zu Unsicherheiten bezüglich Indikationen und ethischer Implikationen im Umgang mit Opioiden auch bei COVID-19 führen.

Ziel der Arbeit: Wir untersuchten bei Mitgliedern der Deutschen Gesellschaft für Palliativmedizin (DGP) die persönliche Wahrnehmung des Umgangs mit Morphin/Opioiden (M/O) zur Symptomkontrolle innerhalb und außerhalb der Palliativmedizin (PM), auch bei der Betreuung COVID-19-Erkrankter.

Material und Methoden: Mittels Survey Monkey ${ }^{\circledR}$ wurden DGP-Mitglieder anonymisiert nach ihrer eigenen Wahrnehmung des Umgangs mit M/O zur Symptomkontrolle befragt.

Ergebnisse und Diskussion: Von den 6192 DGP-Mitgliedern nahmen N=506 teil. Den Umgang mit M/O innerhalb der PM beschrieben $98 \%$ der befragten Ärzt:innen und Pflegekräfte als "sicher und vertraut" bzw. $95 \%$ als „klar geregelt", während dies für die Bereiche außerhalb der PM von weniger als der Hälfte angegeben wurde (48\%/38\%). Bei der Betreuung COVID-19-Erkrankter wurde der Umgang mit M/O außerhalb der PM noch seltener als "sicher und vertraut" (26\%) oder "klar geregelt" (23\%) wahrgenommen. Dyspnoe (99\%/52\%), Erleichterung des Sterbeprozesses (62\%/37\%), Unruhe (30\%/15\%) und Angst/Panik (27\%/13\%) wurden häufiger innerhalb als außerhalb der PM als allgemeine Indikationen genannt. 89\% der Befragten wünschten sich die Einbindung eines PM-Konsilteams.

Schlussfolgerung: Mitglieder der DGP nahmen deutliche Unsicherheiten im Umgang mit M/O außerhalb der PM wahr. Einheitliche interdisziplinäre Leitlinien zur Symptomkontrolle etwa bei Dyspnoe, mehr Lehre und die Einbindung eines PMKonsilteams sollten zukünftig mehr bedacht werden.

\section{Schlüsselwörter}

Opioide $\cdot$ Morphin $\cdot$ COVID-19· Symptomkontrolle $\cdot$ Palliativmedizin

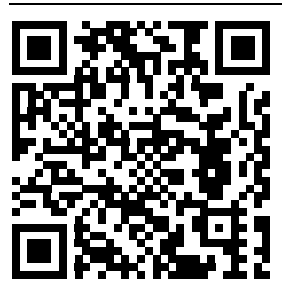

QR-Code scannen \& Beitrag online lesen

\section{Hintergrund und Fragestellung}

Opioide lindern wirksam Schmerzen und Dyspnoe. Leitlinien zu Opioiden bei Dyspnoe divergieren jedoch stark innerhalb von Disziplinen wie Palliativmedizin (PM),
Pneumologie und Intensivmedizin [21, 22, 26, 30]. In der "S3 Leitlinie - Empfehlungen zur stationären Therapie von Patienten mit COVID-19" wurden bis zur dritten Pandemiewelle "Symptomkontrolle", "Morphin" oder "Opioide" nicht erwähnt [20]. Teils 
polarisierende Auffassungen in den Medien über Intensivmedizin und Sterberisiko [11] sowie die Anklage eines Oberarztes wegen Totschlags an COVID-19 erkrankten Patienten [5] veranschaulichen eine teils brisante Situation.

Wir untersuchten daher Wahrnehmungen zum Umgang mit Morphin/Opioiden (M/O) zur Symptomkontrolle innerhalb und außerhalb der PM, allgemein und bei COVID-19.

\section{Studiendesign und Untersuchungsmethoden}

Auf Grundlage von Interviews mit Ärzten und Pflegekräften innerhalb wie außerhalb der Palliativmedizin (Einzelinterviews und Fokusgruppeninterview, nicht publiziert) sowie auf der Grundlage internationaler Literatur identifizierten wir häufige Fragestellungen und Assoziationen zum Umgang mit Opioiden in der Symptomkontrolle. Mitgliedern der Deutschen Gesellschaft für Palliativmedizin (DGP) wurde per E-Mail ein Link zu der anonymisierten Online-Umfrage (Survey Monkey ${ }^{\circledR}$ ) zugesandt mit der Bitte um Beantwortung dieser vom 14.09.-11.10.2020. Der Fragebogen beinhaltete soziodemografische Daten und Fragen zur Wahrnehmung der klinischen Anwendung von Opioiden allgemein und speziell bei COVID-19 Erkrankten.

Wir erläuterten im Fragebogen: „Basierend auf klinischen Erfahrungsberichten sowie internationaler Literatur entsteht der Eindruck, dass der Umgang mit Opioiden wie Morphin zur Symptomkontrolle mit Unsicherheit behaftet sein kann. Daher möchten wir eine Umfrage unter den Mitgliedern der DGP zum Umgang mit Opioiden durchführen. Wir sind an Ihren eigenen Erfahrungen interessiert, aber auch daran, wie Sie Kolleginnen und Kollegen anderer Fachrichtungen im Umgang mit Opioiden zur Symptomlinderung wahrnehmen. [...] „Morphin“ soll dabei exemplarisch für die Gruppe der Opioide genannt werden."

Wir erklärten, dass wir in dieser Untersuchung die Fragen „zwischen dem palliativmedizinischen Bereich (z. B. Palliativstation oder ambulanter Palliativdienst) und der Anwendung in anderen Fachkliniken (z.B. Innere Medizin, Chirurgie,
Orthopädie/Unfallchirurgie, Neurologie, Intensivstationen etc.)" unterscheiden. Diese Unterscheidung bezeichnen wir hier nachfolgend als „innerhalb der PM" und "außerhalb der PM".

Zur Einschätzung setzten wir auch eine sechsstufige Likert-Skala ein (,stimme gar nicht zu“ bis "stimme voll zu“). In der Aufbereitung der Daten wurden die ersten drei Grade rot (,stimme nicht zu“) und die letzten drei Grade blau („stimme zu“) zur Veranschaulichung hinterlegt.

Um die Antworten der Umfrageteilnehmer in Bezug auf innerhalb und auBerhalb der Palliativmedizin zu vergleichen, analysierten wir marginale mittlere Antwortwerte für paarweise Beobachtungen unter Verwendung der Scores 1 bis 6 [2]. Wir präsentieren Schätzungen der Unterschiede der marginalen Mittelwerte und entsprechende $95 \%$-Wald-Konfidenzintervalle. Die zuständige Ethikkommission des Uniklinikums Aachen genehmigte die Durchführung der Studie (EK 303/20).

\section{Ergebnisse}

\section{Drop-out-Analyse}

Von den 6192 DGP-Mitgliedern ${ }^{1}$ nahmen 506 an der Online-Umfrage teil (Antwortrate $8,2 \%)$. Nach ihrer überwiegenden beruflichen Tätigkeit gefragt, gaben 282 Teilnehmer (TN) an, Ärzt:innen zu sein, 164 waren Pflegekräfte, 21 gehörten anderen Berufsgruppen an (4 Psycholog:innen, 6 Sozialarbeiter:innen, 5 Physiotherapeut:innen und 6 Seelsorgende). 37 TN machten keine Angabe.

Ein Vergleich der soziodemografischen Struktur ergab eine vergleichbare Aufteilung von Umfrage-TN zu allen DGPMitgliedern: Männer 33\%/37\%, Frauen $66 \% / 62 \%$; Alter 18-30 Jahre: 3\%/1\%, 31-40 Jahre: 10\%/9\%, 41-50 Jahre: 29\%/23\%, 51-60 Jahre: $42 \% / 38 \%$.

Der Anteil der Berufe bei den Umfrage TN/DGP Mitgliedern war ebenfalls sehr ähnlich: Ärzt:innen 55\%/55\%; Pflege $32 \% / 29 \%$; Soziale Arbeit 1\%/3\%; Psychologie $1 \% / 2 \%$; Seelsorge 1\%/2\%; Physiotherapie $1 \% /$ Physio-Ergo-Logotherapie

\footnotetext{
1 Deutsche Gesellschaft für Palliativmedizin (2020) Persönliche Kommunikation: Mitgliederzahl und-struktur.
}

(letztere beiden in DGP-Datei gemeinsam erfasst) $2 \%$.

\section{Wahrnehmung des Umgangs mit $\mathrm{M} / \mathrm{O}$}

Für Fragen zur klinischen Anwendung von M/O werden in diesem Artikel primär die Daten von Ärzt:innen und Pflegekräften gezeigt, da diese Berufsgruppen Opioide primär anwenden.

Unter den Ärzt:innen und Pflegekräften schätzten den Umgang mit M/O nahezu alle $(98 \%)$ für den PM Bereich als sicher und vertraut ein, jedoch nur zur Hälfte (48\%) für den Bereich außerhalb der PM (• Abb. 1a, Grade 4-6/6). Je älter die Ärzt:innen und Pflegekräfte waren, umso häufiger stimmten sie der Frage nach sicherem Umgang im PM-Bereich „voll zu“ (Grad 6/6) -30 Jahre $60 \%, 31-40$ Jahre $67 \%, 41-50$ Jahre: $73 \%$, 51-60 Jahre:78\%, > 60 Jahre $87 \%$ (nicht in der Abb.).

Betrachtet man die Angaben sämtlicher Umfrage-TN (inklusive Physiotherapeut:innen, Psycholog:innen, Sozialarbeiter:innen), so stimmten diese ebenfalls zu 99\% einem sicheren Umgang innerhalb der PM zu, jedoch für den Bereich außerhalb der PM noch $4 \%$ weniger (gesamte $\mathrm{TN}=44 \%$ ) als bei der Auswertung der Angaben von Ärzt:innen und Pflegekräften. Dabei übersprangen acht TN die Frage (nicht in der Abb.).

\section{Wahrnehmungen zur Klarheit der} Regelung einer M/O-Gabe

Eine klare Regelung für die Gabe von M/O sahen ebenfalls fast alle (95\%) für den $P M-B e r e i c h$, aber nur $38 \%$ für den Bereich außerhalb der PM (- Abb. 2a, Grade 4-6/6).

Bezogen auf COVID-19 Erkrankte beurteilten etwa ein Drittel weniger Ärzt:innen und Pflegekräfte den Umgang mit M/O innerhalb der PM als sicher (65\%) bzw. die Regelung als klar (62\%), aber für den Bereich außerhalb der PM nur jeder vierte als sicher (26\%) bzw. klar geregelt (23\%) (- Abb. 1 b und 2b, jeweils Grade 4-6/6).

Die häufigsten allgemeinen Indikationen für die Gabe von M/O waren "Schmerzen" und "Dyspnoe" (je 99\%) innerhalb der PM (- Abb. 3a), gefolgt von „Erleichterung des Sterbeprozesses" 


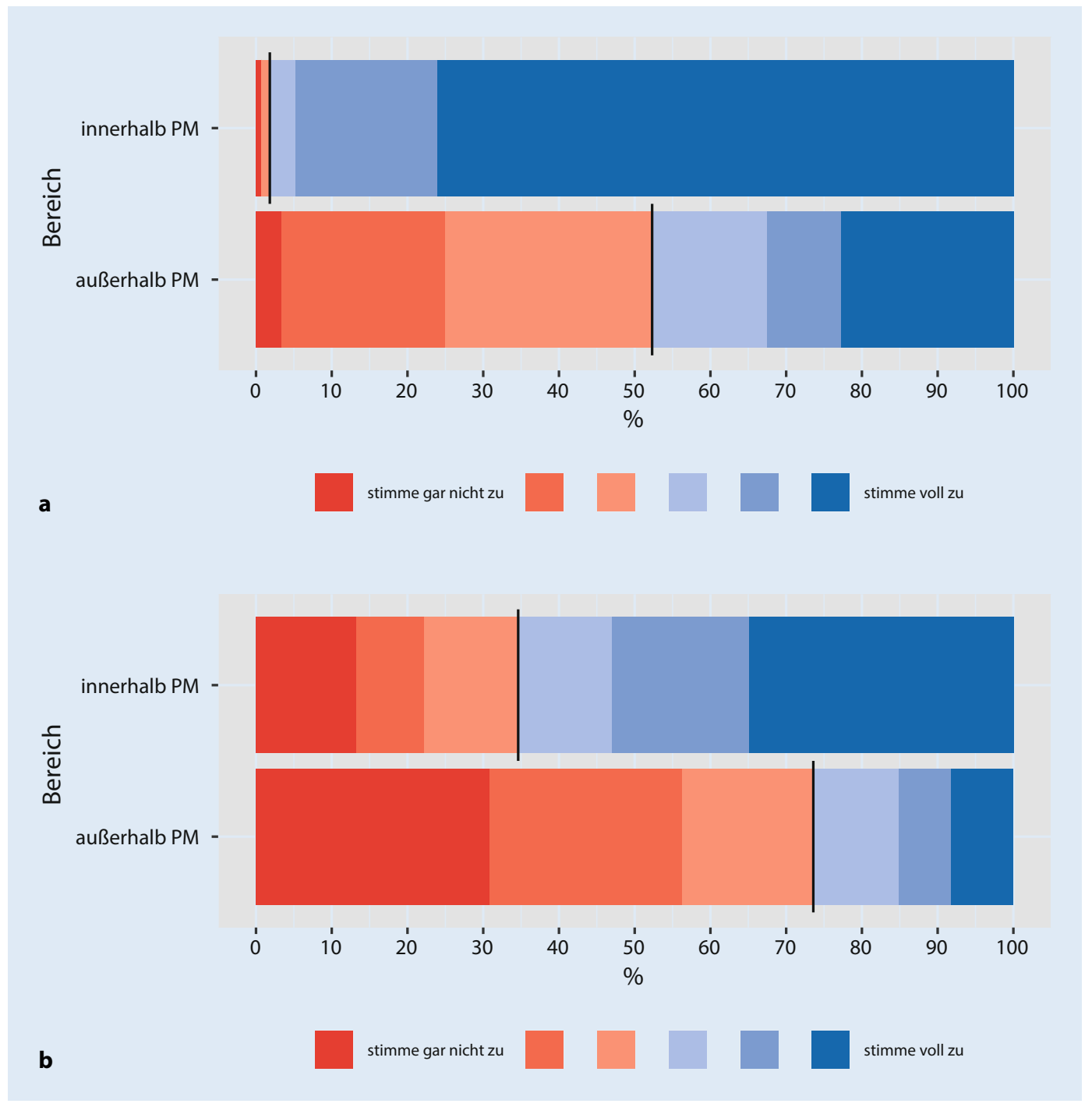

Abb. $1<$ Umgang mit Morphin. a „Der Umgang mit Morphin ist sicher und vertraut." $\mathbf{b}$ "Der Umgang mit Morphin bei COVID-19 Erkrankten ist sicher und vertraut"

(62\%), "Unruhe" (30\%) und "Angst/Panik" (27\%). Erneut schätzte nur etwa die Hälfte der Ärzt:innen und Pflegekräfte ihre Kolleg:innen außerhalb der PM so ein, dass für diese die Symptome "Dyspnoe" (52\%), "Erleichterung des Sterbeprozes-

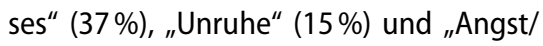
Panik" (13\%) als M/O Indikation gelten würden.

Die Indikationen Dyspnoe, Unruhe, Angst/Panik wurden im Vergleich zum Bereich innerhalb der PM zwar insgesamt seltener außerhalb der PM wahrgenommen, jedoch außerhalb der PM bei COVID19 Erkrankten häufiger als bei den allgemeinen Angaben (- Abb. 3a, b).

Ebenso beurteilten nahezu alle Ärzt:innen und Pflegekräfte (99\%) eine Atemdepression als unerwünschte Wirkung von M/O innerhalb der PM als gut einschätz- bar und kontrollierbar. Jedoch hielten nur etwa die Hälfte (45\%) der Opioidanwendenden diese Atemdepression außerhalb der PM für gut einschätzbar und kontrollierbar (• Abb. 4a).

Bezogen auf COVID-19Erkrankte, wurde jeweils innerhalb und außerhalb der PM etwas häufiger geschätzt, dass Atemdepression eine unerwünschte Wirkung von M/O ist, die beunruhigt (17\%/66\%) (• Abb. 4b).

\section{Wahrnehmung zum Einsatz von M/O beim Sterben}

Die TN Mehrheit negierte, dass M/O innerhalb und außerhalb der PM gezielt eingesetzt werde(n), um das Sterben zu beschleunigen (95\%/83\%). Die meisten der $\mathrm{TN}$, die jedoch zustimmten, gaben dies mit $17 \%$ für den Bereich außerhalb der PM an (ohne COVID-19-Bezug). Außerhalb der PM empfanden nur $10 \%$ der TN den M/O-Einsatz bei der Behandlung COVID19 Erkrankter als Beschleunigung des Sterbens (-Abb. 5a, b, Grade 4-6/6).

Diejenigen TN, die für die Bereiche innerhalb (5\%) wie außerhalb (17\%) der $\mathrm{PM}$ angaben, M/O werde(n) gezielt eingesetzt, um das Sterben zu beschleunigen, waren überwiegend Ärzt:innen, die über 20 Jahre Erfahrung hatten, im Krankenhaus tätig sowie älter als 50 Jahre waren.

Wahrnehmung zu bevorzugten Applikationswegen bei der Anwendung von $\mathrm{M} / \mathrm{O}$

Als bevorzugte Applikationsformen von M/O wurde überwiegend die orale Gabe 


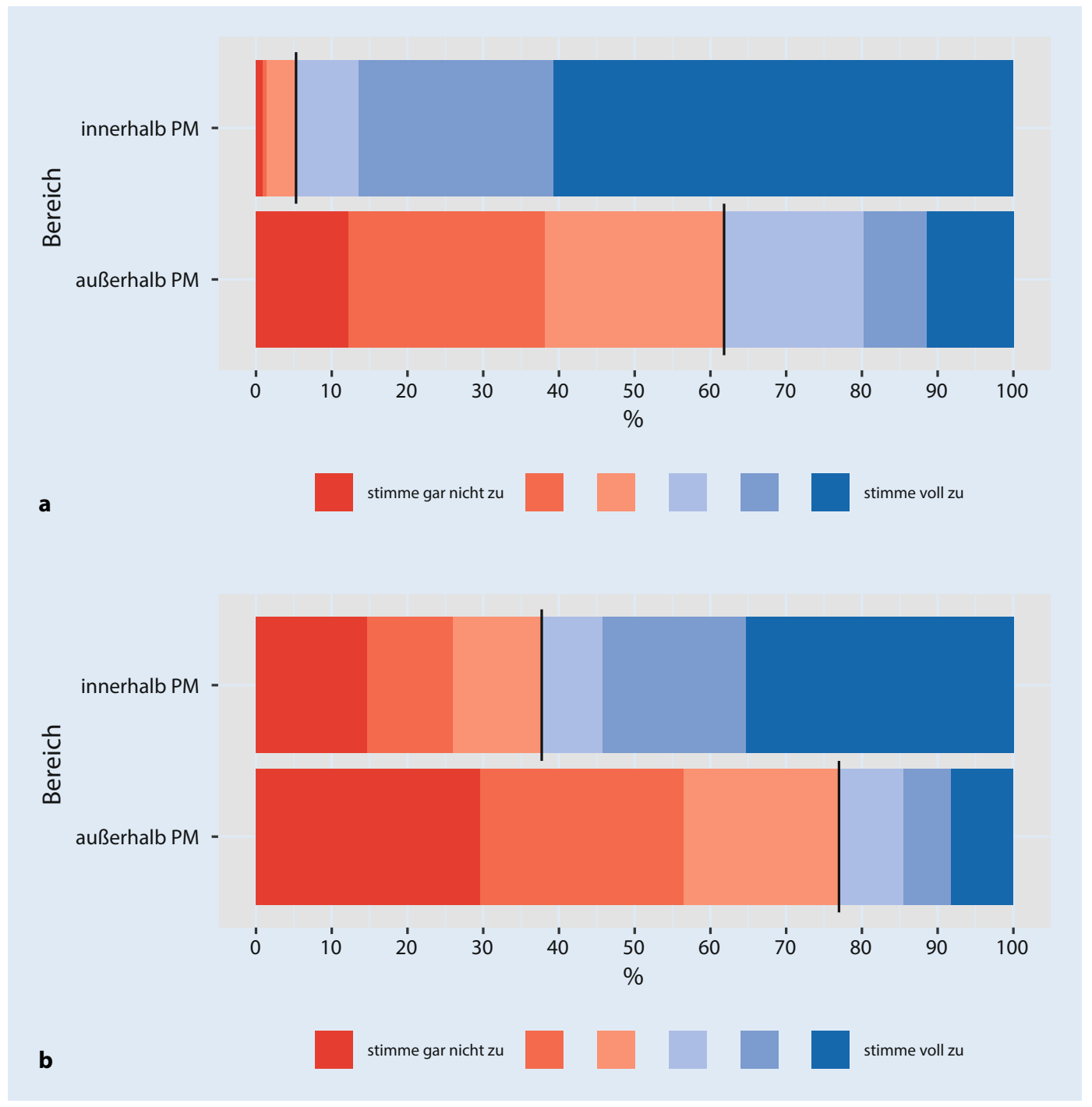

Abb. $2 \triangleleft$ Regelung zur Indikationsstellung für $\mathrm{M} / \mathrm{O}$ Gabe. a "Die Indikation für die Gabe von Morphin ist klar und einheitlich gere-

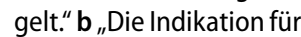
die Gabe von Morphin bei COVID-19 Erkrankten ist klar und einheitlich geregelt"

angegeben (innerhalb/außerhalb PM: $80 \% / 76 \%$ ), gefolgt von intravenös (iv, $51 \% / 37 \%$ ), subkutan (sc, $88 \% / 36 \%$ ) und transdermal $(47 \% / 49 \%)$.

Gewünscht wurde von allen TN allgemein vor allem die "(bessere) Einbindung eines palliativmedizinischen Konsilteams" (89\%), gefolgt von "(mehr) Lehre (77\%)", "(mehr) Teamkonferenzen" (76\%) und "(mehr) Supervisionen" (71\%). Hier wurde nicht zwischen den Bereichen innerhalb und außerhalb der PM unterschieden.

\section{Vergleich der Bereiche innerhalb versus außerhalb der PM}

Vergleicht man die Wahrnehmungen für die Bereiche innerhalb versus außerhalb der PM, so stimmten die TN eher für den Bereich innerhalb der PM zu im Hinblick auf folgende Fragen: „Die Indikation für die Gabe von Morphin ist klar und einheitlich geregelt" (Unterschied des Durchschnittswerts [innerhalb der PM - außerhalb der PM] 2,2; $95 \%$ Konfidenzintervall 2,1; 2,4) und "Der Umgang mit Morphin ist sicher und vertraut" $(1,9 ; 1,8 ; 2,1)$.

Diese Unterschiede fielen etwas schwächer bei den Fragen mit COVID-19 Bezug aus, aber weiterhin stimmten TN eher für den Bereich innerhalb der PM zu, verglichen mit außerhalb der PM: „Die Indikation für die Gabe von Morphin bei COVID19 Erkrankten ist klar und einheitlich geregelt" $(1,5 ; 1,3 ; 1,7)$, und "Der Umgang mit Morphin bei COVID-19 Erkrankten ist sicher und vertraut" $(1,5 ; 1,4 ; 1,7)$.

Vergleicht man die Fragen "Morphin wird gezielt eingesetzt um das Sterben zu beschleunigen" $(-0,6 ;-0,7 ;-0,4)$ und
"Morphin wird bei COVID-19 Erkrankten gezielt eingesetzt um das Sterben zu beschleunigen" $(-0,3 ;-0,4 ;-0,2)$, so stimmten die TN eher für den Bereich außerhalb der PM zu.

\section{Diskussion}

\section{Heterogener Stellenwert von Opioiden in Leitlinien}

Opioide sind für den Einsatz bei starken Schmerzen zugelassen [6], während die Anwendung bei Dyspnoe off-label ist. Die palliativmedizinische S3-Leitlinie für Menschen mit nicht mehr heilbarer Krebserkrankung empfiehlt Opioide klar zur Therapie von u. a. Schmerz und Dyspnoe (Atemnot) [22]. 


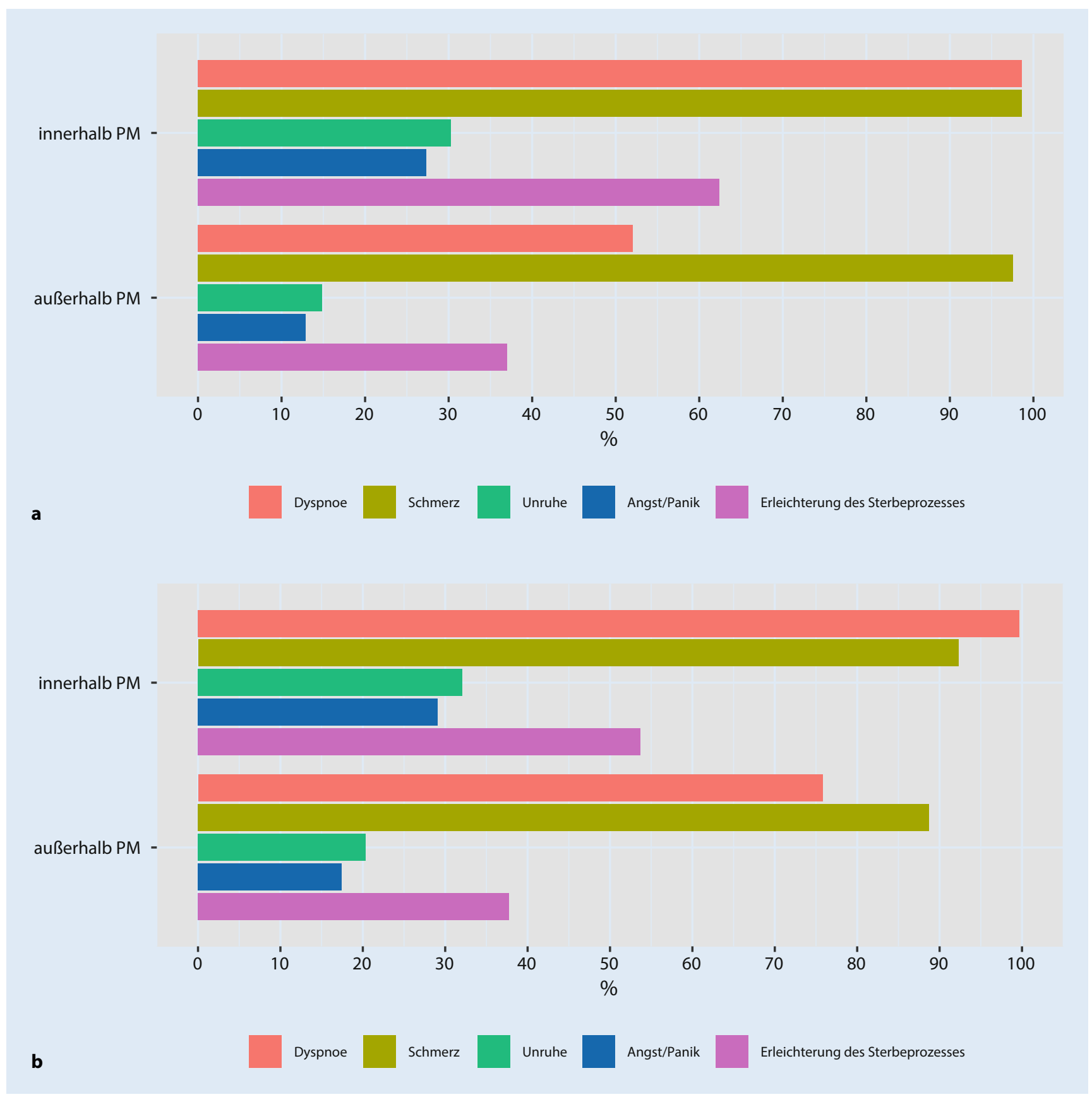

Abb. 3 \ Allgemeine Indikationen für M/O Gabe. a „Allgemeine Indikationen für die Gabe von Morphin sind“ b „Indikationen für die Gabe von Morphin bei der Betreuung COVID-19 Erkrankter sind“

Während Rosenbruch et al. in ihrer „SOP - Atemnot bei erwachsenen Patienten" bei fehlendem Ansprechen auf allgemeine, nichtpharmakologische Maßnahmen ebenfalls die Gabe von Opioiden und ggf. Benzodiazepinen empfehlen [28], sind andere Leitlinien zur Symptomkontrolle von Dyspnoe mit Opioiden zurückhaltend formuliert [30] oder nennen Opioide hierzu gar nicht [20, 21]: In der „S3-Leitlinie - Empfehlungen zur stationären Therapie von Patienten mit COVID-19“ wurde Palliativmedizin bis zum 16.05.2021 vor allem im Sinne von Therapiebegrenzung genannt [20]. Zwar wurde festgestellt: „Der Palliativversorgung mit dem Ziel der optimalen Linderung von belastenden Symptomen wie Dyspnoe, Husten, Schwäche und Fieber, Angst, Panik, Unruhe und Delir kommt in diesen Situationen eine besondere Bedeutung zu“, jedoch fehlten konkrete Empfehlun- gen zur Symptomkontrolle mit Opioiden wie sie in separaten Handlungsempfehlungen der Deutschen Gesellschaft für Palliativmedizin dargestellt waren [26]. Dosierungsbeispiele zur Symptomkontrolle mit Opioiden fanden in die S3Leitlinie erst während der dritten COVID19 Welle am 17.05.2021 Eingang, dann unter Beteiligung der Deutschen Gesellschaft für Palliativmedizin [19]. 


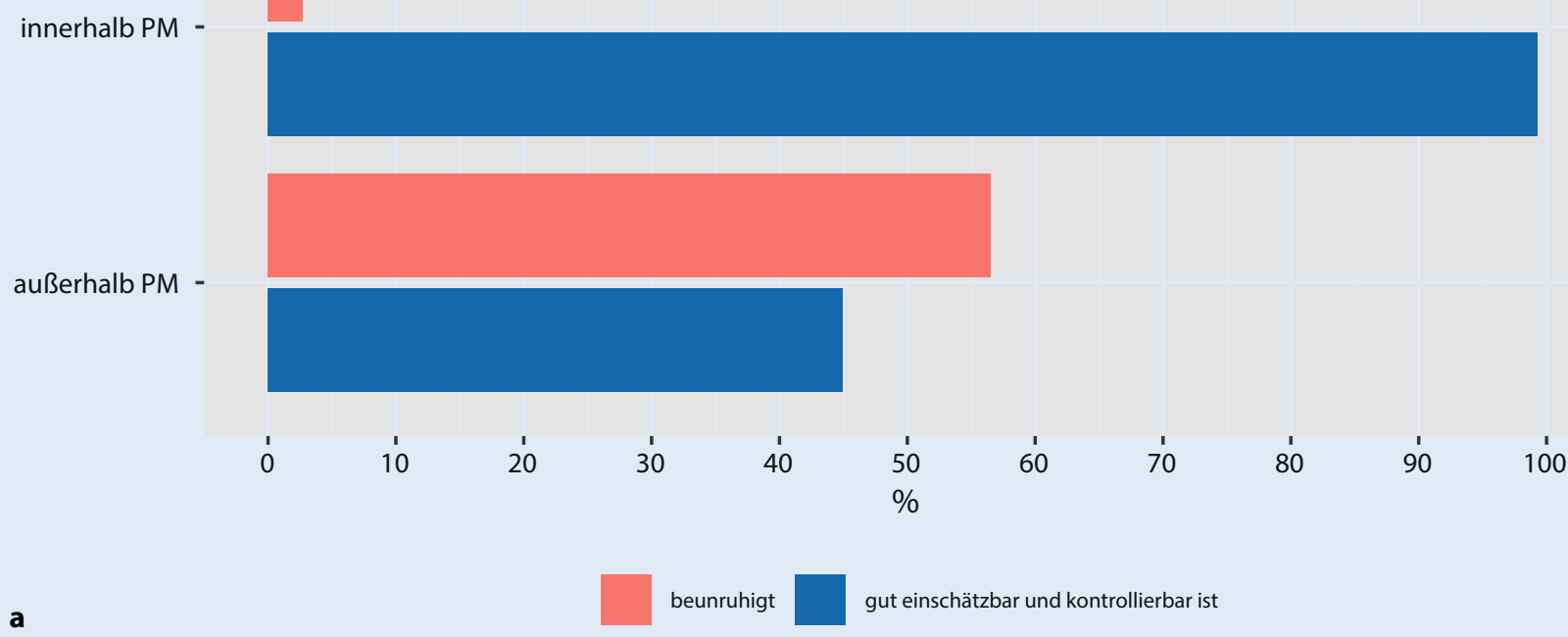

innerhalb PM -

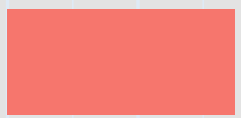

außerhalb PM -
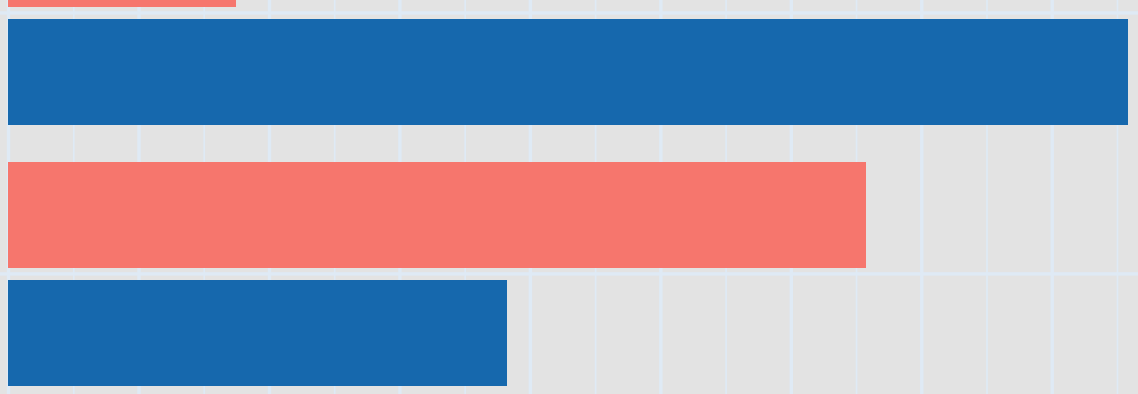

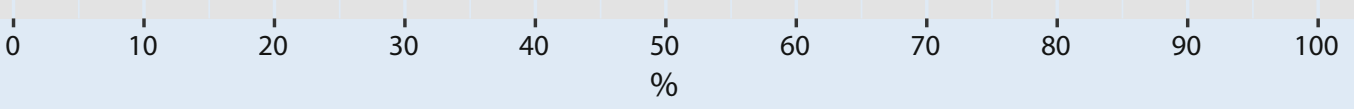

b

beunruhigt

gut einschätzbar und kontrollierbar ist

Abb. 4 \ Einschätzung von Atemdepression als unerwünschte Wirkung. a „Atemdepression ist eine unerwünschte Wirkung

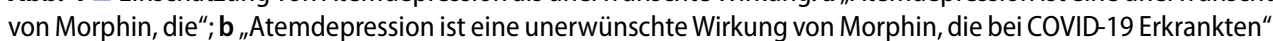

Die im Jahr 2005 publizierten „Empfehlungen zur Behandlung respiratorischer Komplikationen bei einer Viruspandemie" der Deutschen Gesellschaft für Pneumologie nannten Opioide nicht zur Symptomkontrolle der Dyspnoe, sondern nur bezüglich "stärkerer Schmerzen" und der "Einleitung der Beatmung" [21]. Die Empfehlungen der aktuelleren COPDLeitlinie warnen vor „bedeutsame(n), unerwünschte(n) Effekten, insbesonde- re der Atemdepression“, weswegen „der Einsatz auf wenige besonders beeinträchtigte Patienten mit schwerer Atemnot beschränkt" sein solle [30]. Ein aktuelles systematisches Review zur Behandlung von Atemnot bei Patient:innen mit fortgeschrittener Krebserkrankung fand keine Assoziation zwischen pharmakologischen Substanzen und erhöhter Wirksamkeit gegenüber Placebo [15]. Die Autoren bewerteten viel der Evidenz mit „niedriger
Qualität" und betonten, dass zukünftige Studien Atemnot als multidimensionales Geschehen messen sollten.

\section{Stellenwert von Opioiden bei Dyspnoe und COVID-19}

Die Diagnose und Therapie von Luftnot und Ateminsuffizienz sind komplex [8]. Die medizinische Versorgung überwiegend älterer Patient:innen mit teils lebenslimitie- 


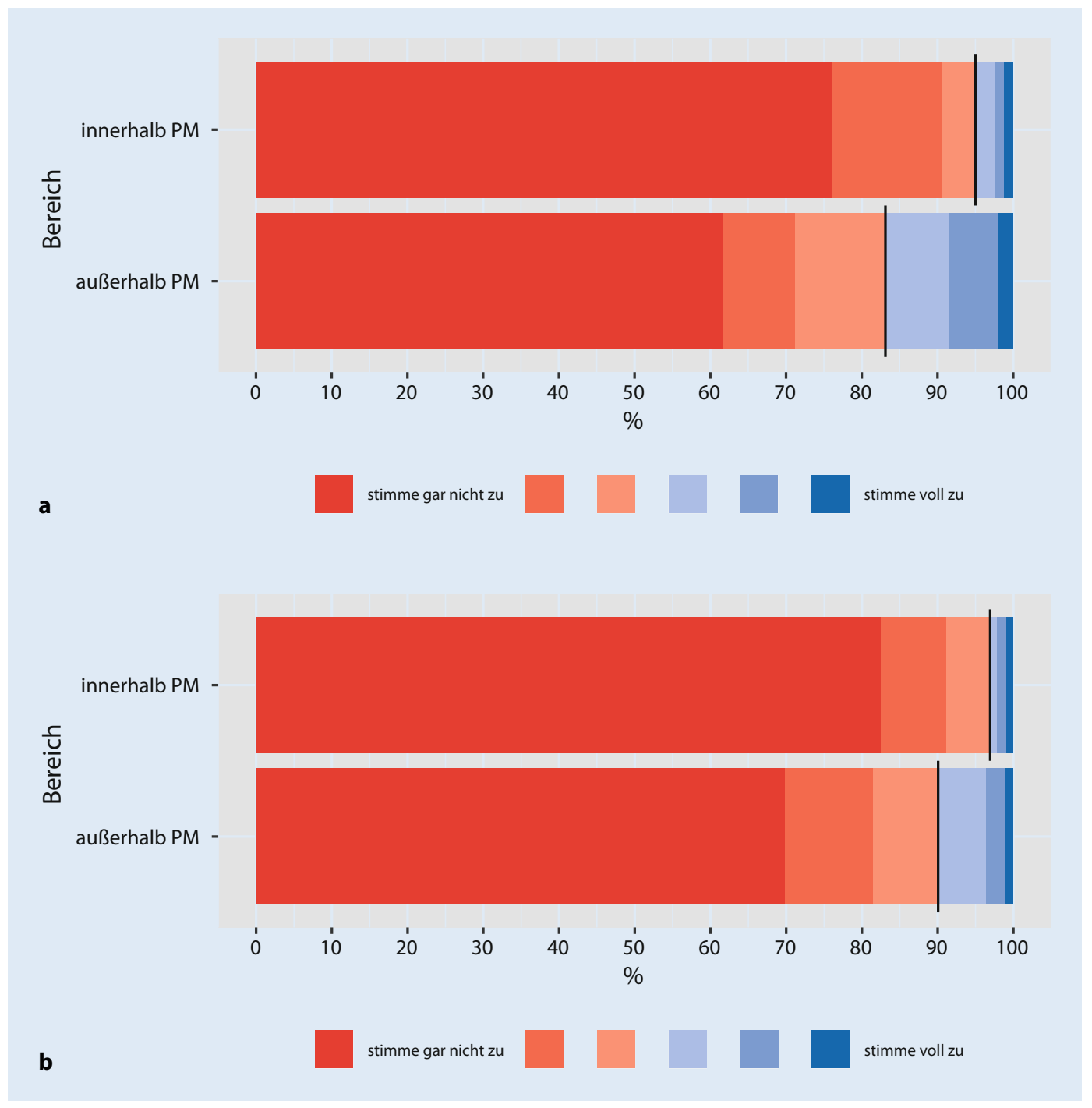

Abb. 5 - Einsatz von M/O beim Sterben. a "Morphin wird gezielt eingesetzt, um das Sterben zu beschleunigen." b "Morphin wird bei COVID-19 Erkrankten gezielt eingesetzt, um das Sterben zu beschleunigen"

renden Komorbiditäten beinhaltet internistische und intensivmedizinische Interventionen bis hin zu Intubation und Beatmung. Dabei nimmt die angemessene Symptombehandlung, insbesondere von Luftnot, eine Schlüsselrolle ein $[3,7,14$, 17, 23, 26]. Palliativmedizinisch betreute Patienten mit COVID-19 zeigten in der aktuellen Literatur insbesondere Dyspnoe und Agitation als führende Symptome, die meist erfolgreich mit relativ niedrig dosierten Opioiden und Benzodiazepinen kontrolliert werden konnten [3, 17, 23]. Der Grad der Dyspnoe schien unabhängig vom Grad einer Hypoxämie aufzutreten [3]. Weitere Symptome beinhalteten Schmerz, Angst und Übelkeit, während Husten kaum oder gar nicht auftrat. Eine typische Opioiddosis in den letzten $24 \mathrm{~h}$ des Lebens waren $15 \mathrm{mg}$ Morphin sub- kutan, kontinuierlich über einen Perfusor verabreicht [16]. Der Einsatz von Opioiden erscheint also effizient bereits in niedrigen Dosierungen, auch bei COVID-19, und im Sinne einer adäquaten Symptomkontrolle wichtig für die Patient:innen.

\section{Große Unterschiede innerhalb und außerhalb der Palliativmedizin in unserer Umfrage}

Ärzt:innen und Pflegekräfte betrachteten den Umgang mit $\mathrm{M} / \mathrm{O}$, innerhalb der PM auch beim Thema Atemdepression, als sicherer im Vergleich zu außerhalb der PM. Dieser Trend zeigte sich ebenfalls bei Unruhe, Angst/Panik, und der Erleichterung des Sterbeprozesses. Ein besonders großer Unterschied bestand für die Dyspnoe: Nur die Hälfte der TN meinte, dass M/O zur
Behandlung von Dyspnoe außerhalb der PM eingesetzt werden würde.

Diese Unsicherheit, insbesondere im außerpalliativmedizinischen Bereich, könnte assoziiert sein mit der Inkonsistenz der o.g. Leitlinien außerhalb der Palliativmedizin.

Ein deutlicher Warnhinweis war die Bewertung bei $17 \%$ der TN, dass Morphin außerhalb der PM gezielt eingesetzt werde, um das Sterben zu beschleunigen (-Abb. 5a). Allerdings kann dabei nicht differenziert werden zwischen einer Intention der Beschleunigung im Sinne von aktiver Sterbehilfe oder nur eine Inkaufnahme eines potenziell schnelleren Versterbens im Rahmen der Symptomkontrolle. Die Inkaufnahme nach dem Prinzip des "doppelten Effekts" wäre in der ethischen Bewertung akzeptabel, 
wenn z. B. Morphin zur Symptomkontrolle eingesetzt wird, dabei aber auch das Risiko akzeptiert wird, dass dadurch zu einem früheren Versterben beigetragen werden könnte [9]. Eine beabsichtigte Beschleunigung wäre hingegen ethisch und juristisch nicht akzeptabel. Allerdings ist bei einer sachgerechten und individuell angepassten Titration mit Opioiden bei sterbenden Menschen mit Schmerzen nicht zu erwarten, dass sie vorzeitig an einer solchen Opioidgabe versterben.

Eine retrospektive Studie stationär eingewiesener Patienten, die eine reine Symptomkontrolle und Änderung der Opioiddosis erhielten, zeigte keinen signifikanten Unterschied bezüglich der Zeit bis zum Versterben in der Niedrigdosis- versus Hochdosisgruppe [1]. Das Einbeziehen einer palliativmedizinischen Konsultation hingegen erhöhte die Überlebenszeit sogar signifikant in dieser Studie. Dies weist darauf hin, dass ein schnelleres Versterben durch Opioidgabe unwahrscheinlich ist.

\section{Ideen zur Verbesserung der Versorgung}

Prinzipiell sind Vorbehalte gegenüber dem Einsatz von Opioiden ein weltweit bekanntes Phänomen, das zu einer Unterversorgung von Patienten führen kann [7]. Dazu kommen Aspekte wie Assoziation von Morphin mit Abhängigkeit, Sterben [10] und dem Nicht-Aushalten von Leid [29]. Da in der SARS-CoV-2-Pandemie eine starke psychische Belastung des medizinischen Personals besteht, kann die Konstellation aus Vorbehalten, Unsicherheiten und auch heterogenen Leitlinien verschiedener Fachgesellschaften die medizinische Versorgung und angemessene Symptomkontrolle von Patienten mit COVID-19 zusätzlich erschweren. Die Unvorhersagbarkeit des Verlaufs einer COVID-19-Erkrankung stellt eine weitere Unsicherheit dar. Ein Teil der COVID-19-Erkrankten leidet unter Dyspnoe, während diese bei anderen fehlt $[3,17,23]$. Eine adäquate Einschätzung der betroffenen Patienten ist also per se eine klinische Herausforderung und muss aufgrund des dynamischen Verlaufs dieser Erkrankung immer wieder neu erfolgen.

Die beschriebenen Unterschiede können durchaus eine Versorgungslücke für Patient:innen mit Luftnot und insbeson- dere auch mit COVID-19 bedeuten. Einen Ansatz, diese Versorgungslücke zu schließen, stellt die Entwicklung evidenzbasierter, einheitlicher und fachübergreifender Leitlinien dar, um Widersprüchlichkeiten oder fachabhängige „blinde Flecken“ zu vermeiden. Im Bereich der Onkologie und PM gibt es bereits umfassende Empfehlungen für die Therapie der Dyspnoe (hier: „Atemnot ${ }^{\prime \prime}$ ), die z.B. allgemeine mit nichtpharmakologischen Maßnahmen und Therapien der behandelbaren Ursachen kombinieren $[22,28]$. Opioide haben hier trotz des Off-Label-Einsatzes einen relevanten Stellenwert.

Eine individuelle und wirksame Symptomkontrolle stellt eine der vielen Herausforderungen einer Pandemie dar. Palliativmediziner:innen fühlten sich in unserer Umfrage im Umgang mit M/O sicher, beurteilen diesen Umgang für nicht palliativmedizinische Fachbereiche jedoch als deutlich unsicherer: Dieses Ergebnis untermauert die Bedeutung des Einbeziehens eines palliativmedizinischen Konsildienstes, der auch von den meisten Umfrage-TN gewünscht wurde und in der Literatur empfohlen wird [23]. Im ambulanten Bereich könnten Teams der allgemeinen und spezialisierten Palliativversorgung als Ansprechpartner:innen zur Verfügung stehen. Fadul et al. [14] fordern hierzu explizit eine Integration von Palliativmediziner:innen in die COVID-19-PandemiePlanung. Deren Einbindung in Entscheidungsalgorithmen und in Schulungsmaßnahmen zur Symptomkontrolle sollten dabei im Vordergrund stehen, ebenso die Begleitung des medizinischen Personals. Sie betrachten die Erhaltung der Arbeitsfähigkeit vor dem Hintergrund psychisch belastender Situationen und Entscheidungen als vorrangig.

Darüber hinaus müssen Aus, Fortund Weiterbildungsmaßnahmen kurz-, aber auch langfristig die Vermittlung von Kenntnissen und Fertigkeiten im Umgang mit Opioiden noch klarer berücksichtigen $[13,25]$. Kausale und symptomatische Therapieansätze - vor allem mit Opioiden können sowohl bei kurativen als auch bei palliativen Behandlungsabsichten auch kombiniert gegen Luftnot zum Einsatz kommen. Dabei ist die subkutane Applikationsform eine einfache und wirksame Methode zur Gabe von Opioiden. Auch in unserer Umfrage fiel auf, dass die subkutane Gabe mit $89 \%$ innerhalb der PM versus $36 \%$ außerhalb der PM mehr als doppelt so häufig genannt wurde. Gerade bei Patienten in der letzten Lebensphase kann diese Applikation vorteilhaft sein.

\section{Zusätzliche Erkenntnisse zum Umgang mit $\mathrm{M} / \mathrm{O}$}

Unruhe, Angst/Panik und Erleichterung des Sterbeprozesses sind keine anerkannten Indikationen für Opioide [6], auch wenn sich in der Literatur Hinweise für einen sinnhaften Einsatz über die klassischen Indikationen hinaus finden: $z$. B. bei Agitation, die ein Zeichen für Schmerzen sein kann [18]. Eine nicht-indikationsgemäße Anwendung schnell freisetzender Fentanyl-Formulierungen war vielen TN schmerzmedizinischer Fortbildungen in einer stichprobenartigen Fragebogenerfassung wie folgt bekannt: „im eigenen Patientengut" 25\%, "bei mitbehandelnden Kollegen" 25\%, "berichtsweise" $40 \%$ [31].

Auch wird in der Literatur diskutiert, ob Morphin bei einem Teil der COVID-19-Erkrankten Stress reduzieren kann [27]. Hier sollten im klinischen Setting in ergänzende und alternative Konzepte wie Benzodiazepine zur Behandlung von Dyspnoe und Unruhe erörtert werden. Studien zur Frage, inwieweit Opioide sich womöglich prognostisch auf das Immunsystem bei COVID-19-Erkrankten auswirken könnten, wären zukünftig wünschenswert. Ein im Jahr 2020 publiziertes systematisches Review zu Patienten mit chronischen NichtKrebsschmerzen stellte lediglich mit einer "sehr schwachen Evidenz" von "niedriger Qualität" fest, dass sich eine LangzeitOpioidbehandlung auf das Immunsystem auswirken könnte [12]. Aufgrund fehlender Konsistenz in der Outcome-Erfassung war keine Metaanalyse möglich.

\section{Limitationen}

Die Rücklaufquote war mit $8,2 \%$ relativ niedrig, und die Ergebnisse sollten daher vorsichtig interpretiert werden. Niedrige Beteiligungen an Online-Umfragen werden häufig berichtet [4]. So war der häufigste Grund für eine Nichtteilnahme unter Allgemeinmedizinern deren Eindruck, 
Hier steht eine Anzeige.

黑 Springer 
von zu vielen Umfragen überschwemmt zu werden, und die Problematik begrenzter Zeitressourcen [24].

Anhand einer Drop-out-Analyse auf der Basis vorhandener Paramater in der DGP Datenbank - wie der Berufs- und AItersstruktur der DGP Mitglieder - konnten wir eine gute Vergleichbarkeit herleiten und halten daher eine Repräsentativität der Stichprobe für naheliegend. Für valide Ergebnisse spricht, dass die Erkenntnisse dieser Studie die Ergebnisse z.B. der qualitativen Studie von Charalambous et al. ergänzen, in denen die Unsicherheit im Umgang mit Opioiden deutlich wurde: Ärzt:innen und Patient:innen assoziierten Morphin mit Tod/Erkrankung im Endstadium und Abhängigkeit [10].

Schwierig einzuschätzen ist, inwieweit das Verständnis einiger Formulierungen unterschiedlich interpretiert worden ist: Der Bereich „innerhalb der PM“ könnte z. B. das eigene PM Team betreffen, aber auch andere PM-Bereiche, während "auBerhalb der PM" sich auf sämtliche andere Fachbereiche bezieht und sehr heterogen verstanden worden sein kann. Zusammengefasst sollte die Bereichszuordnung als grobe Einschätzung eingeordnet werden. Darüber hinaus sind die Aussagen für den Bereich außerhalb der PM nur indirekt über die Mitglieder der Palliativgesellschaft erhoben worden.

Morphin wurde im Fragebogen stellvertretend für Opioide genannt (s.a. „Studiendesign und Untersuchungsmethoden“). Diese Festlegung wurde It. Rückmeldungen einzelner TN unterschiedlich wahrgenommen, da es an einigen Kliniken teils etablierte Konzepte mit anderen Opioiden gibt. Es könnte also sein, dass die konkrete Nennung nur dieses einen Opioids zu einer womöglich leicht veränderten Beantwortung führte.

Wir hatten Morphin gewählt, da es unserer Einschätzung nach das am häufigsten angewandte Opioid in der Klinik sowie in der hier referierten internationalen Literatur ist. So sollte für die Umfrage-TN ein konkreter Bezug zu konkreten Situationen im Klinik-/Praxisalltag leichter hergestellt werden können.

In den Fragen zu • Abb. 4a, b wurden unbeabsichtigt Mehrfachnennungen im Online-Fragebogen nicht ausgeschlossen, sodass zur Frage der • Abb. 4a 9 bzw.
6 TN und bei - Abb. 4b 15 bzw. 12 TN doppelte Angaben machten. Dies hat jedoch nur zu diskreten Änderungen der Häufigkeiten geführt.

\section{Fazit für die Praxis}

- Die Mitglieder der DGP nahmen ein hohes Maß an Unsicherheit im Umgang mit Opioiden zur Symptomkontrolle außerhalb der PM wahr, insbesondere bezüglich des Umgangs mit M/O bei COVID-19Erkrankten. Ihren eigenen Bereich schätzten sie bezüglich der Klarheit zum Einsatz von Opioiden als sicher ein und sahen inn als gut geregelt an. Die Ergebnisse bestätigen die von den Teilnehmern vorgebrachte Notwendigkeit einer stärkeren Einbindung der spezialisierten Palliativeinrichtungen in die Planungen und Organisation der Behandlung von Patienten mit Covid-19.

- Die Entwicklung evidenzbasierter, einheitlicher und fachübergreifender Leitlinien ist erforderlich, um eine bestmögliche Versorgung COVID-19-Erkrankter unter Einbindung von palliativmedizinisch erfahrenen Behandelnden zu gewährleisten.

Korrespondenzadresse

Dr. med. Vera Peuckmann-Post, PhD

Klinik für Palliativmedizin, Medizinische

Fakultät, RWTH Aachen University

Pauwelsstr 30, 52074 Aachen, Deutschland vpeuckmann@ukaachen.de

Danksagung. Wir bedanken uns bei der Deutschen Gesellschaft für Palliativmedizin für die Unterstützung bei der praktischen Durchführung der Umfrage. Vera Peuckmann-Post wurde gefördert durch das Landesprogramm für chancengerechte Hochschulmedizin des Ministeriums für Kultur und Wissenschaft des Landes Nordrhein-Westfalen.

Funding. Landesprogramm für chancengerechte Hochschulmedizin des Ministeriums für Kultur und Wissenschaft des Landes Nordrhein-Westfalen

Funding. Open Access funding enabled and organized by Projekt DEAL.

\section{Einhaltung ethischer Richtlinien}

Interessenkonflikt. V. Peuckmann-Post, A. Scherg, N. Krumm, C. Hagedorn, A. Keszei und F. Elsner geben an, dass kein Interessenkonflikt besteht. L. Radbruch war Präsident der Deutschen Gesellschaft für Palliativmedizin bis Februar 2021. R. Rolke ist Sprecher der Sektion Ärztinnen und Ärzte der Deutschen Gesellschaft für Palliativmedizin sowie der klinischen Kommission für neurologische Palliativmedizin der Deutschen Gesellschaft für Neurologie. Er erhielt For- schungsförderung vom G-BA Innovationsfonds, BMBF, der Robert-Bosch-Stiftung, RWTH Aachen und PallPan (Initiative des Netzwerks Universitätsmedizin). Prof. Rolke erhielt Honorare für Vorträge oder wissenschaftliche Beratertätigkeiten von Aristo Pharma, Eli Lilly and Company, Grünenthal Deutschland $\mathrm{GmbH}$, Spectrum Therapeutics, Tilray Deutschland GmbH.

Die zuständige Ethikkommission des Uniklinikums Aachen genehmigte die Durchführung der Studie (EK 303/20).

Open Access. Dieser Artikel wird unter der Creative Commons Namensnennung 4.0 International Lizenz veröffentlicht, welche die Nutzung, Vervielfältigung, Bearbeitung, Verbreitung und Wiedergabe in jeglichem Medium und Format erlaubt, sofern Sie den/die ursprünglichen Autor(en) und die Quelle ordnungsgemäß nennen, einen Link zur Creative Commons Lizenz beifügen und angeben, ob Änderungen vorgenommen wurden.

Die in diesem Artikel enthaltenen Bilder und sonstiges Drittmaterial unterliegen ebenfalls der genannten Creative Commons Lizenz, sofern sich aus der Abbildungslegende nichts anderes ergibt. Sofern das betreffende Material nicht unter der genannten Creative Commons Lizenz steht und die betreffende Handlung nicht nach gesetzlichen Vorschriften erlaubt ist, ist für die oben aufgeführten Weiterverwendungen des Materials die Einwilligung des jeweiligen Rechteinhabers einzuholen.

Weitere Details zur Lizenz entnehmen Sie bitte der Lizenzinformation auf http://creativecommons.org/ licenses/by/4.0/deed.de.

\section{Literatur}

1. Abraham DL, Hernandez I, Ayers GT et al (2021) Association between opioid dose escalation and time to death in a comfort measures only population. Am J Health Syst Pharm 78:203-209

2. Agresti A (2010) Analysis of ordinal categorical data. Wiley, Hoboken

3. Alderman B, Webber K, Davies A (2020) An audit of end-of-life symptom control in patients with corona virus disease 2019 (COVID-19) dying in a hospital in the United Kingdom. Palliat Med 34:1249-1255

4. Alt-Epping B, Bauer J, Schuler U et al (2014) Pain therapyin oncology: results of a nationwide survey. Schmerz 28:157-165

5. Deutsches Ärzteblatt (2021) Oberarzt angeklagt: Tödliche Arzneimittel für drei COVID-19-Patienten. https://www.aerzteblatt.de/nachrichten/123197/ Oberarzt-angeklagt-Toedliche-Arzneimittelfuer-drei-COVID-19-Patienten; Zugegriffen: 16. Juli 2021

6. Rote Liste Service GmbH (2021) Rote Liste

7. Bandieri E, Potenza L, Efficace F et al (2020) Independent research on cancer pain management in the setting of early palliative care: a flywheel to counteract general opioid misuse and abuse. Int J Environ Res Public Health 17(19):7097

8. Berliner D, Schneider N, Welte T et al (2016) The differential diagnosis of dyspnea. Dtsch Arztebl Int 113:834-845

9. Brescia FJ (2003) Philosophical oncology: calling on the principle of double effect. J Natl Compr Canc Netw 1:429-434 
10. Charalambous A, Zorpas M, Cloconi C et al (2019) Healthcare professionals' perceptions on the use of opioid analgesics for the treatment of cancerrelated pain in Cyprus: a mixed-method study. SAGE Open Med 7:2050312119841823

11. Deutsche Gesellschaft Für Anästhesiologie und Intensivmedizin (DGAl) (2020) Anästhesisten kritisieren WDR-Berichterstattung zu Beatmung - „Völlig unnötige und unqualifizierte Emotionalisierung eines wichtigen intensivmedizinischen Themas" - Einzelmeinung als Fachempfehlung dargestellt. https://www.dgai.de/pressemitteilungen/ pressemitteilungen/682-anaesthesistenkritisieren-wdr-berichterstattung-zu-beatmungvoellig-unnoetige-und-unqualifizierteemotionalisierung-eines-wichtigen-intensiv medizinischen-themas-einzelmeinung-als-fachempfehlung-dargestellt.html;. Zugegriffen: 30. Apr. 2020

12. Diasso PDK, Birke H, Nielsen SD et al (2020) The effects of long-term opioid treatment on the immune system in chronic non-cancer pain patients:asystematicreview. Eur JPain 24:481-496

13. Elsner F, Centeno C, Ellershaw JE (2016) Early integration needs early education. Palliat Med 30:805-806

14. Fadul N, Elsayem AF, Bruera E (2021) Integration of palliative care into COVID-19 pandemic planning. BMJ Support Palliat Care 11(1):40-44

15. Feliciano JL, Waldfogel JM, Sharma R et al (2021) Pharmacologic interventions for breathlessness in patients with advanced cancer:a systematic review and meta-analysis. JAMANetw Open 4:e2037632

16. Heath L, Carey M, Lowney AC et al (2021) Pharmacological strategies used to manage symptoms of patients dying of COVID-19: a rapid systematic review. Palliat Med 35:1099-1107

17. Hetherington $L$, Johnston $B$, Kotronoulas $G$ et al (2020) COVID-19 and hospital palliative care-a service evaluation exploring the symptoms and outcomes of 186 patients and the impact of the pandemic on specialist hospital palliative care. Palliat Med 34:1256-1262

18. Husebo BS, Ballard C, Sandvik R et al (2011) Efficacy of treating pain to reduce behavioural disturbances in residents of nursing homes with dementia: cluster randomised clinical trial. BMJ 343:d4065

19. Kluge $S$, Janssens $U$, Welte T, Weber-Carstens $S$ et al (2021) S3-Leitlinie - Empfehlungen zur stationären Therapie von Patienten mit COVID-19. AWMF-Register-Nr. 113/001

20. Kluge $S$, Janssens $U$, Welte $T$, Weber-Carstens $S$ et al (2020) S3-Leitlinie - Empfehlungen zur stationären Therapie von Patienten mit COVID-19. AWMF-Register-Nr. 113/001

21. Köhler D, Karg O, Lorenz J et al (2005) Recommendations for the treatment of respiratory complications in case of a viruspandemic. Pneumologie 59:720-724

22. Leitlinienprogramm Onkologie (Deutsche Krebsgesellschaft, Deutsche Krebshilfe, AWMF) (2020) Palliativmedizin für Patienten mit einer nicht-heilbaren Krebserkrankung, Langversion 2.1. AWMFRegisternummer: 128/001-OL

23. Lovell N, Maddocks M, Etkind SN et al (2020) Characteristics, symptom management and outcomes of 101 patients with COVID-19 referred forhospital palliative care.JPain Symptom Manage 60(1):e77-e81

24. Macpherson I, Bisset A (1995) Not another questionnaire!: Eliciting the views of general practitioners. Fam Pract 12:335-338

\section{Perceptions on the use of opioids in COVID-19. A survey of members of the German Association for Palliative Medicine}

Background: Opioids efficiently alleviate pain and dyspnea. However, guidelines on symptom management with opioids differ, which may lead to an uncertainty concerning opioid indication and ethical implication among medical staff, especially when caring for COVID-19 patients.

Aims: We aimed to examine the perception of members of the German Association for Palliative Medicine (DGP) concerning the administration of morphine as the gold standard opioid (subsequently termed $\mathrm{M} / \mathrm{O}$ ) for symptom control within and outside of a palliative care (PC) setting, including care for COVID-19 patients.

Methods: DGP members received an anonymized online questionnaire (Survey Monkey ${ }^{\circledR}$ ) containing questions regarding their perception of symptom management with $\mathrm{M} / \mathrm{O}$ in general and in particular concerning COVID-19 patients. Participants were asked to rate their perception within and outside of a PC setting.

Results: Of the 6129 DGP members, $\mathrm{N}=506$ participated. DGP physicians and nurses perceived handling of M/O as "certain and confident" (98\%) and "clearly regulated" within PC (95\%) but rated it significantly lower for outside PC (48\%/38\%). When caring for COVID-19 patients, handling of M/O was even less often rated "certain and confident" (26\%) or "clearly regulated" (23\%) for outside PC. Dyspnea $(99 \% / 52 \%)$, relief from the dying process $(62 \% / 37 \%)$, restlessness $(30 \% / 15 \%)$ and fear or panic $(27 \% / 13 \%)$ were more frequently rated as general indications for morphine within versus outside PC. Most participants (89\%) wished to involve palliative care consultation teams.

Conclusions: DGP members perceived substantial uncertainty in the handling of M/O for medical fields outside PC. Uniform interdisciplinary guidelines for symptom control, more education, and involvement of a PC consultation team should be increasingly considered in the future.

\section{Keywords}

Opioid · Morphine · COVID-19 · Symptom control · Palliative care

25. Mason S, Paal P, Elsner F et al (2020) Palliative care for all: an international health education challenge. Palliat Support Care 18(6):760-762

26. Nehls W, Delis S, Haberland B et al (2020) Handlungsempfehlung zur Therapie von Patient*innen mit COVID-19 aus palliativmedizinischer Perspektive 2.0. AWMF, Berlin

27. Pfeifer M, Ewig S, Voshaar T et al (2020) Position paper for the state of the art application of respiratory support in patients with COVID19-German respiratory society. Pneumologie 74(6):337-357

28. Rosenbruch J, Eschbach C, Viehrig M et al (2018) SOP - Atemnot bei erwachsenen Palliativpatienten. Onkologe 24:18-21

29. StreeckN (2020) Death without distress? The taboo of suffering in palliative care. Med Health Care Philos 23:343-351

30. VogelmeierCEA (2018) S2k-Leitlinie zur Diagnostik und Therapie von Patienten mit chronisch obstruktiver Bronchitis und Lungenemphysem (COPD)

31. Wirz S, Schenk M, Hofbauer H et al (2021) Use of rapid-onset fentanyl preparations beyond indication: a random questionnaire survey among congress participants and pain physicians. Schmerz 35:114-123 